\title{
HEISENBERG UNCERTAINTY INEQUALITY FOR GABOR TRANSFORM
}

\author{
ASHISH BANSAL AND AJAY KUMAR
}

Abstract. We discuss the Heisenberg uncertainty inequality for groups of the form $K \ltimes \mathbb{R}^{n}, K$ is a separable unimodular locally compact group of type I. This inequality is also proved for Gabor transform for several classes of groups of the form $K \ltimes \mathbb{R}^{n}$.

Mathematics subject classification (2010): Primary 43A32; Secondary 43A30, 22D10, 22D30, 22E25. Keywords and phrases: Heisenberg uncertainty inequality, Fourier transform, Gabor transform, Heisenberg group, nilpotent Lie group, Plancherel formula.

\section{REFERENCES}

[1] A. Baklouti And N. B. Salah, On theorems of Beurling and Cowling-Price for certain nilpotent Lie groups, Bull. Sci. Math. 132 (2008), 529-550.

[2] A. BANSAL AND A. KUMAR, Generalized analogs of the Heisenberg uncertainty inequality, Journal of Inequalities and Applications 2015:168, Springer (2015), 1-15.

[3] P. Ciatti, M. G. Cowling And F. Ricci, Hardy and uncertainty inequalities on stratified Lie groups, Advances in Mathematics 277, Elsevier (2015), 365-387.

[4] P. CiatTi,F. RiCCI AND M. SUNDARI, Heisenberg-Pauli-Weyl uncertainty inequalities and polynomial volume growth, Advances in Mathematics 215 (2), Elsevier (2007), 616-625.

[5] A. G. FARASHAHI AND R. KAMYABI-Gol, Continuous Gabor transform for a class of non-Abelian groups, Bull. Belg. Math. Soc. 19, Simon Stevin (2012), 683-701.

[6] G. B. Folland, A Course in Abstract Harmonic Analysis, CRC Press (1994).

[7] G. B. Folland and A. Sitaram, The Uncertainty Principle: A Mathematical Survey, The Journal of Fourier Analysis and Applications 3 (1997), 207-238.

[8] E. Kaniuth And A. Kumar, Hardy's Theorem for simply connected nilpotent Lie groups, Math. Proc. Camb. Phil. Soc. 131 (2001), 487-494.

[9] A. KLEPPNER AND R. LiPSMAn, The plancherel formula for group extensions II, Annales scientifiques de l'École Normale Supérieure 6 (1), (1973), 103-132.

[10] G. W. MACKEY, The theory of unitary group representations, University of Chicago Press Chicago (1976).

[11] O. A. Nielson, Unitary representations and coadjoint orbits of low-dimensional nilpotent Lie groups, Queens Papers in Pure and Appl. Math., Queen's Univ., Kingston, ON, (1983).

[12] A. Sitaram, M. Sundari And S. ThangaVelu, Uncertainty principles on certain Lie groups, Proc. Math. Sci. 105 (1995), 135-151.

[13] K. Smaoui, Beurling's Theorem for nilpotent Lie groups, Osaka J. Math. 48 (2011), 127-147.

[14] S. Thangavelu, Some uncertainty inequalities, Proc. Indian Acad. Sci. 100 (2), (1990), 137-145.

[15] ElKe WILCZOK, New uncertainty principles for the continuous Gabor transform and the continuous wavelet transform, Documenta Mathematica 5, Universiät Bielefeld, Fakultät für Mathematik (2000), 201-226.

[16] J. XiAO AND J. HE, Uncertainty inequalities for the Heisenberg group, Proc. Indian Acad. Sci. (Math. Sci.) 122 (4), (2012), 573-581. 